\title{
INVENTARIO DEL CONOCIMIENTO TAXONÓMICO, ASÍ COMO DE LA DIVERSIDAD Y DEL ENDEMISMO REGIONALES DE LAS ESPECIES MEXICANAS DE BURSERA (BURSERACEAE)*
}

\author{
Jerzy Rzedowski ${ }^{1}$, Rosalinda Medina Lemos ${ }^{2}$ y \\ Graciela CaLderón de Rzedowski ${ }^{1}$ \\ ${ }^{1}$ Instituto de Ecología, A.C., Centro Regional del Bajío, \\ Apartado postal 386, 61600 Pátzcuaro, Michoacán \\ ${ }^{2}$ Instituto de Biología, Universidad Nacional Autónoma de México, \\ Apartado postal 70-233, 04510 México, D.F.
}

\section{RESUMEN}

El conocimiento de la sistemática de Bursera es aún manifiestamente incompleto. En el presente trabajo se ofrece una lista comentada de 82 especies mexicanas de este género que ameritan al menos un reconocimiento provisional, indicando para cada una la distribución geográfica conocida por estados de la República.

Con base en esta información se elabora un mapa y un cuadro de diversidad de los representantes de Bursera en las 32 entidades administrativas del país. Guerrero registra 48 especies, seguido por Michoacán y Oaxaca con 37, así como por Jalisco y Puebla con 28 respectivamente. En Tlaxcala no se ha encontrado ninguna, mientras que Nuevo León, Tabasco y Quintana Roo albergan sólo una cada uno.

Se integra asimismo una lista de 33 especies mexicanas de Bursera ( $40.3 \%$ del total) de distribución restringida, 10 de las cuales se registran únicamente de la localidad tipo y áreas aledañas, 10 adicionales se conocen aparentemente de un solo estado y otras 13 no se extienden más allá del territorio de dos estados contiguos. Con fundamento en estos datos se definen seis áreas de concentración de especies endémicas del género, ubicándolas en el mapa respectivo.

Palabras clave: Bursera, Burseraceae, diversidad, endemismo, México.

\footnotetext{
*Trabajo realizado con apoyo económico del Instituto de Ecología, A. C. (cuenta 902-07), del Consejo Nacional de Ciencia y Tecnología y de la Comisión Nacional para el Conocimiento y Uso de la Biodiversidad.
} 


\section{ABSTRACT}

Our knowledge concerning the systematics of Bursera is still very imperfect. In this paper we present an annotated list of 82 Mexican species of this genus that merit at least a provisional recognition, indicating for every species its known geographical distribution on the state level.

On the basis of this information a map and a table are presented showing the diversity of Bursera in the 32 Mexican administrative divisions. Fourty eight species are reported for Guerrero, 37 for Michoacán and Oaxaca, and 28 for Jalisco and Puebla. Tlaxcala has no recorded species, while Nuevo León, Tabasco and Quintana Roo shelter only one species each.

A list of 33 Mexican species of Bursera with restricted distribution $40.3 \%$ of the total) is also presented, 10 being reported only from the type locality and its surroundings, 10 additional ones known only from one state, and 13 extending their areas into two contiguous states. On the basis of these data six areas are defined and mapped, in which endemic species of the genus are known to concentrate.

Key words: Bursera, Burseraceae, diversity, endemism, Mexico.

\section{INTRODUCCIÓN}

Bursera es un miembro prominente y característico tanto de la flora como de la vegetación de México. Se trata, sin embargo, de un grupo cuyo conocimiento aún deja bastante que desear, pues a su sistemática complicada se une la circunstancia de que la existencia de muchas de sus especies no se ha descubierto o discernido correctamente todavía (para una discusión más detallada sobre el particular véase el trabajo de Rzedowski et al., 2004).

En 1984 Kohlmann y Sánchez Colón realizaron un análisis areográfico de los componentes mexicanos del género y con ello marcaron una serie de patrones de repartición de este grupo de plantas. Para su estudio los mencionados autores examinaron la distribución geográfica de 64 especies de Bursera en el territorio de la República.

De entonces a la fecha se han descrito 14 taxa adicionales y se revalorizaron otros cuatro, de suerte que en la actualidad cabe reconocer la existencia de alrededor de 80 representantes más o menos bien entendidos de este conjunto de la flora nacional. Dado, a su vez, el insuficiente grado de exploración botánica de amplios sectores del país, se puede conjeturar que la real cuantía de los componentes de Bursera de México rebasa la cifra de 100. No hay duda de que la región costera entre Jalisco y Oaxaca, la adyacente Sierra Madre del Sur, así como algunos rincones de la cuenca del Balsas, están escondiendo todavía un significativo contingente de especies locales de este género. 
El gran caudal de nuevas colectas obtenidas en las últimas décadas ha permitido también ampliar el registro y el entendimiento de la repartición geográfica y ecológica de las plantas en cuestión, aunque manifiestamente en este ámbito quedan aún numerosas lagunas por llenar.

El propósito principal de la presente contribución consiste en:

a) poner al día la lista de las especies mexicanas de Bursera más o menos claramente comprendidas;

b) registrar la distribución geográfica hasta la fecha conocida de cada uno de tales taxa a nivel de presencia o ausencia en los estados de la República, y con ello ofrecer una más avanzada aproximación al patrón de la diversidad regional del género;

c) analizar los casos de las especies de área notablemente restringida y ubicar los sectores de concentración de los endemismos estrechos.

\section{ENUMERACIÓN DE LAS ESPECIES}

Se enlistan a continuación las especies mexicanas de Bursera que ameritan al menos una aceptación provisional en el estado actual del conocimiento. Para cada una de ellas se anexa un comentario, los sinónimos más importantes, así como la indicación relativa a la literatura en que es factible encontrar descripciones aceptablemente completas y las ilustraciones correspondientes. Se define asimismo su distribución geográfica conocida, tomando como criterio las 32 divisiones administrativas principales de nuestro país.

De las 82 especies incluidas, 42 (las de los números $1,3,4,5,6,8,12,15,16$, $18,21,23,24,28,30,32,34,41,43,44,45,46,49,50,52,53,54,56,57,58,59,60$, $62,66,69,70,71,74,75,77,78$ y 79) pertenecen a la sección (o subgénero) Bursera. Las restantes 40 (de los números, 2, 7, 9, 10, 11, 13, 14, 17, 19, 20, 22, 25, 26, 27, 29, $31,33,35,36,37,38,39,40,42,47,48,51,55,61,63,64,65,67,68,72,73,76,80$, 81 y 82) se adscriben a su vez a la sección Bullockia (o subgénero Elaphrium).

1. Terebinthus acuminata Rose (no Bursera acuminata Willd.), planta del complejo de $B$. simaruba, cuyas relaciones con $B$. attenuata, así como la distribución geográfica más precisa quedan todavía pendientes de aclararse. Dadas estas circunstancias, carece aún de binomio apropiado en Bursera. Frecuentemente presente en situaciones transicionales con encinares y pinares en las cuencas de Lerma-Santiago y del Balsas.

Descripción disponible en Toledo Manzur (1982).

Conocida con cierta seguridad de: Ags., Col., Gro., Jal., Méx., Mich., Nay., Zac. 
2. B. altijuga Rzed., Calderón \& Medina. Endemismo estrecho de la región limítrofe entre Puebla y Oaxaca. Planta por lo general arbustiva que forma parte de algunos matorrales xerófilos en altitudes superiores a $1700 \mathrm{~m}$. Descripción e ilustración disponibles en Rzedowski et al. (2004).

Distribución geográfica conocida: Oax., Pue.

3. B. aptera Ramírez. Taxon relativamente bien definido del complejo de $B$. fagaroides. Abundante en la cuenca del Papaloapan y en la parte oriental de la del Balsas. Descripción disponible en Ramírez (1896), Toledo Manzur (1982), Guízar Nolazco y Sánchez Vélez (1991) y en Rzedowski et al. (2004); el primero y los últimos dos trabajos también incluyen una ilustración.

Distribución geográfica conocida: Gro., Mor., Oax., Pue.

4. B. arborea (Rose) Riley. Taxon aproximadamente bien definido del complejo de $B$. simaruba. Habitante de comarcas próximas al litoral del Pacífico, en altitudes inferiores a $1000 \mathrm{~m}$. Descripción disponible en Toledo Manzur (1982).

Distribución geográfica conocida: Col., Dgo., Gro., Jal., Mich., Nay., Sin., posiblemente también presente en Oax.

5. B. arida (Rose) Standl. Endemismo de la región limítrofe entre Puebla y Oaxaca. Planta a menudo arbustiva, habitante de matorrales xerófilos. Descripción e ilustración disponibles en Rose (1906) y en Rzedowski et al. (2004).

Distribución geográfica conocida: Oax., Pue.

6. B. ariensis (H.B.K.) McV. \& Rzed. (B. brachypoda (Rose) Engl., B. pannosa Engl., $B$. sessiliflora Engl.). Componente bien definido y de amplia distribución del complejo de $B$. fagaroides. Habitante preferente de altitudes superiores a $1000 \mathrm{~m}$, a menudo en áreas de transición con los encinares. Descripción disponible en Toledo Manzur (1982) y en Rzedowski et al. (2004); las ilustraciones pueden consultarse en el último trabajo, así como en McVaugh y Rzedowski (1965).

Distribución geográfica conocida: Chis., Gro., Jal., Méx., Mich., Mor., Nay., Oax., Pue.

7. B. aspleniifolia Brandegee. Endemismo estrecho y poco frecuente de la región limítrofe entre Puebla y Oaxaca. Especie cercanamente relacionada con $B$. copallifera y en algunas áreas donde las dos conviven, se han observado señales de introgresión recíproca. Descripción e ilustración disponibles en Rzedowski et al. (2004).

Distribución geográfica conocida: Oax., Pue. 
8. B. attenuata (Rose) Riley. Componente del complejo de B. simaruba, aún no muy bien esclarecido, sobre todo frente a Terebinthus acuminata, por lo que tampoco está bien definida su área. Planta de la Sierra Madre Occidental y de comarcas de la planicie costera del Pacífico. Descripción escueta disponible en Rose (1909).

Distribución geográfica conocida: Dgo., Nay., Sin.

9. B. bicolor (Willd. ex Schltdl.) Engl. Elemento propio de la cuenca del Balsas y también de la del río Tehuantepec. Notable por la coloración muy diferente del haz y del envés de las hojas. Descripción disponible en Schlechtendal (1843), en Engler (1883), en Toledo Manzur (1982) y en Guízar Nolazco y Sánchez Vélez (1991); este último trabajo incluye una ilustración.

Distribución geográfica conocida: Gro., Méx., Mich., Mor., Oax., Pue.

10. B. biflora (Rose) Standl. Endemismo del norte de Oaxaca y de áreas adyacentes de Puebla, donde es habitante frecuente del bosque tropical caducifolio y del matorral xerófilo. Especie morfológicamente muy variable. Descripción e ilustración disponibles en Rzedowski et al. (2004).

Distribución geográfica conocida: Oax., Pue.

11. B. bipinnata (DC.) Engl. (B. elemifera (Royle) Baill., B. gracilis Engl.). Una de las especies de más amplia repartición y de las más comunes. Es también la que con más frecuencia produce individuos híbridos con varios otros miembros de la sección Bullockia. Tales híbridos se identifican a menudo con el nombre de $B$. diversifolia Rose, por lo que este último binomio no se acepta formalmente como perteneciente a un taxon en particular. Descripciones disponibles en Schlechtendal (1843), Toledo Manzur (1982), Guízar Nolazco y Sánchez Vélez (1991), Rzedowski y Guevara-Féfer (1992), Rzedowski y Calderón de Rzedowski (1996b) y en Rzedowski et al. (2004); todos, salvo los dos primeros trabajos, incluyen ilustración, misma que también puede consultarse en Engler (1931).

Distribución geográfica conocida: Ags., Chis., Col., Dgo., Gto., Gro., Jal., Méx., Mich., Mor., Nay., Oax., Pue., Ver., Zac.

12. B. bolivarii Rzed. Componente satisfactoriamente diferenciado del complejo de B. fagaroides. Especie no muy común de la porción oriental de la cuenca del Balsas. Descripción disponible en Rzedowski (1968) y en Toledo Manzur (1982); el primer trabajo incluye también una ilustración.

Distribución geográfica conocida: Gro., Oax., Pue. 
13. B. bonetii Rzed. Endemismo aparentemente estrecho de la parte central de Guerrero. En el extremo occidental de Oaxaca se han colectado muestras de una planta similar, pero está pendiente la decisión de si realmente pertenece a la misma especie. Descripción disponible en Rzedowski (1968) y en Toledo Manzur (1982); el primer trabajo también incluye una ilustración.

Distribución geográfica conocida: Gro.

14. B. cerasifolia Brandegee. Endemismo estrecho del extremo sur de la península de Baja California. A menudo desarrollándose en forma de arbusto bajo en lugares próximos a la orilla del mar. Descripción disponible en Wiggins (1964); las ilustraciones pueden consultarse en Wiggins (1980) y en Roberts (1989).

Distribución geográfica conocida: B.C.S.

15. B. chemapodicta Rzed. \& Ortiz. Endemismo estrecho del centro de Guerrero. Especie relacionada con B. schlechtendalii. Descripción e ilustración disponibles en Rzedowski y Ortiz (1982).

Distribución geográfica conocida: Gro.

16. B. cinerea Engl. (B. subpubescens (Rose) Engl.). Taxon relativamente bien definido del complejo de $B$. simaruba, endémico de la vertiente del Golfo de México. Descripción e ilustración disponibles en Rzedowski y Calderón de Rzedowski (1996a, 1996b) y en Rzedowski et al. (2004).

Distribución geográfica conocida: Oax., Pue., Ver.

17. B. citronella McVaugh \& Rzed. Habitante del sector occidental de la Sierra Madre del Sur, en altitudes inferiores a $1100 \mathrm{~m}$. Árbol con aroma penetrante y fino. Descripción disponible en McVaugh y Rzedowski (1965) y en Toledo Manzur (1982); el primer trabajo incluye también una ilustración.

Distribución geográfica conocida: Col., Gro., Jal., Mich.

18. B. confusa (Rose) Engl. Especie endémica del extremo sur de Jalisco y de lugares adyacentes de Michoacán, posiblemente también presente en Colima. Descripción escueta disponible en Rose (1911); la ilustración de una hoja puede consultarse en McVaugh y Rzedowski (1965).

Distribución geográfica conocida: Jal., Mich.

19. B. copallifera (DC.) Bullock. (B. jorullensis H.B.K., B. palmeri var. glabrescens S. Wats.). Árbol común y distribuido en las cuencas del Santiago y del Balsas, así como a lo largo de la Sierra Madre del Sur. Descripción disponible en Toledo Manzur (1982) y en Guízar Nolazco y Sánchez Vélez (1991); las 
ilustraciones pueden consultarse en este último trabajo, así como en Engler (1931, como B. jorullensis), en McVaugh y Rzedowski (1965) y en Humboldt, Bonpland y Kunth (1825), en este último bajo el nombre de Elaphrium jorullense.

Distribución geográfica conocida: Col., Gro., Jal., Méx., Mich., Mor., Nay., Oax., Pue., Zac.

20. B. coyucensis Bullock. Especie endémica de la parte occidental de la Depresión del Balsas, donde prospera en altitudes inferiores a $500 \mathrm{~m}$. Descripción disponible en Bullock (1936) y en Toledo Manzur (1982).

Distribución geográfica conocida: Gro., Mich.

21. B. crenata P. G. Wilson. Especie de distribución limitada al sector occidental de la Depresión del Balsas, donde crece en altitudes inferiores a $700 \mathrm{~m}$. Descripción disponible en Wilson (1958) y en Toledo Manzur (1982).

Distribución geográfica conocida: Gro., Mich., Jal.

22. B. cuneata (Schltdl.) Engl. Árbol distribuido en la cuenca del Balsas y en el sector central del Eje Volcánico Transversal, a menudo en altitudes superiores a 2000 m. Descripción disponible en Schlechtendal (1843), en Engler (1883), en Toledo Manzur (1982), así como en Rzedowski y Guevara-Féfer (1992); el último trabajo incluye también una ilustración.

Distribución geográfica conocida: D.F., Gto., Gro., Hgo., Méx., Mich., Mor., Pue., Qro.

23. B. denticulata McVaugh \& Rzed. Especie endémica del sector occidental de la Sierra Madre del Sur y de la cuenca del Balsas, donde prospera en altitudes inferiores a $700 \mathrm{~m}$. Descripción disponible en McVaugh y Rzedowski (1965) y en Toledo Manzur (1982); el primer trabajo incluye también una ilustración.

Distribución geográfica conocida: Col., Gro., Jal., Mich.

24. B. discolor Rzed. Especie endémica de la Depresión del Balsas y de la Sierra Madre del Sur. Miembro bien definido del complejo de B. fagaroides, destacando por la corteza exfoliante de las ramillas. Descripción disponible en Rzedowski (1968) y en Toledo Manzur (1982); el primer trabajo también incluye una ilustración.

Distribución geográfica conocida: Col., Gro., Méx., Mich., Mor., Oax., Pue.

25. B. epinnata (Rose) Engl. (B. nesopola I. M. Johnst.). Arbusto o arbolito propio de la península de Baja California y también presente en las Islas Revillagigedo, de donde fue descrito como B. nesopola I. M. Johnst.; sin embargo, 
no es seguro de que se trate de una especie diferente de $B$. hindsiana. Descripción disponible en Wiggins (1964); una ilustración puede consultarse en Roberts (1989).

Descripción geográfica conocida: B.C., B.C.S., Col.

26. B. esparzae Rzed., Calderón \& Medina. Especie sólo conocida de Oaxaca; es algo frecuente en la Sierra Madre del Sur y se ha colectado en una localidad de la cuenca del Papaloapan. Descripción e ilustración disponibles en Rzedowski et al. (2004).

Distribución geográfica conocida: Oax.

27. B. excelsa (H.B.K.) Engl. (B. sphaerocarpa Sprague \& Riley). Planta de amplia distribución a lo largo de las partes bajas de la vertiente pacífica y también en la Depresión Central de Chiapas. Es el elemento medular de un complejo de varios taxa, cuyos límites no están aún claros. En particular hace falta definir la circunscripción correcta de la especie y de sus variedades frente a $B$. tomentosa (Jacq.) Triana \& Planch., a algunas poblaciones de B. palmeri y de otras plantas cuya identidad se desconoce todavía. Descripción disponible en Toledo Manzur (1982) y en Pennington y Sarukhán (1998); la ilustración correspondiente puede consultarse en esta última publicación y en McVaugh y Rzedowski (1965).

Distribución geográfica conocida: Chis., Col., Dgo., Gro., Jal., Mich., Nay., Oax., Sin.

28. B. fagaroides (H.B.K.) Engl. (B. obovata Turcz., B. schaffneri S. Wats.). Elemento central de un complejo de taxa que requiere de un profundo estudio y de la revalorización de varios de sus componentes. McVaugh y Rzedowski (1965) distinguieron para B. fagaroides tres entidades subespecíficas: var. fagaroides, var. purpusii y var. elongata. Sin embargo, observaciones más recientes muestran que las dos primeras no en todas partes pueden diferenciarse claramente, y con respecto a la tercera, estudios de secuencias de ADN realizadas por Becerra y Venable (1999) y por Becerra (2003) señalan que se trata de una especie independiente. En tal virtud será probablemente necesario reivindicar el nombre de $B$. odorata Brandegee y posiblemente algunos otros, pues queda por definirse el alcance y la repartición geográfica de plantas de esta afinidad, que se conocen desde el SW de Estados Unidos hasta el Istmo de Tehuantepec. En espera de tan complicadas decisiones taxonómicas, a continuación se opta por dar la distribución de la especie en su sentido amplio. Descripciones disponibles en Toledo Manzur (1982), en Guízar Nolazco y Sánchez Vélez (1991), en Rzedowski y Guevara-Féfer (1992), en Johnson (1992) y en Rzedowski et al. (2004); las ilustraciones correspondientes pueden consultarse en el segundo, cuarto y último trabajos, así como en Humboldt, Bonpland 
y Kunth (1824, bajo el nombre de Elaphrium fagaroides), en Engler (1931) y en McVaugh y Rzedowski (1965).

Distribución geográfica conocida: Ags., B.C., B.C.S., Chih., Coah., Col., D.F., Dgo., Gto., Gro., Hgo., Jal., Méx., Mich., Mor., Nay., Oax., Pue., Qro., S.L.P., Sin., Son., Tamps., Ver., Zac.

29. B. filicifolia Brandegee. Taxon endémico de la porción meridional de la península de Baja California. Algunos autores consideran a esta planta como subespecie de B. laxiflora. Descripción disponible en Wiggins (1964); una ilustración puede consultarse en Roberts (1989).

Distribución geográfica conocida: B.C.S.

30. B. fragilis S. Wats. Endemismo del segmento septentrional de la Sierra Madre Occidental. Varios autores consideraron a esta planta como sinónimo taxonómico de $B$. lancifolia; sin embargo recientes trabajos realizados por Becerra y Venable (1999) y por Becerra (2003), basados en el estudio de secuencias de ADN, indican que se trata de una especie independiente. Descripción e ilustración disponibles bajo el nombre de B. lancifolia en Johnson (1992).

Distribución geográfica conocida: Chih., Dgo., Sin., Son.

31. B. fragrantissima Bullock. Elemento endémico de la cuenca del Balsas, que descuella por su aroma intenso y fino. Descripción disponible en Bullock (1937) y en Toledo Manzur (1982).

Distribución geográfica conocida: Gro., Mich.

32. B. galeottiana Engl. Especie de distribución disyunta, separada por el Eje Volcánico Transversal. Es particularmente frecuente en varios sectores de Oaxaca y Puebla, reapareciendo en el área limítrofe de Guanajuato y Querétaro. Descripción e ilustración disponibles en Rzedowski y Guevara-Féfer (1992), así como en Rzedowski et al. (2004).

Distribución geográfica conocida: Gto., Gro., Oax., Pue., Qro.

33. B. glabrifolia (H.B.K.) Engl. (B. nelsonii Rose, B. schiedeana Engl.). Elemento frecuente en la cuenca del Balsas, pero extendiéndose también a las del Papaloapan y del río Tehuantepec, donde prospera en altitudes superiores a 1200 $\mathrm{m}$, a menudo en colindancia con encinares y pinares. Descripción disponible en Toledo Manzur (1982), en Guízar Nolazco y Sánchez Vélez (1991) y en Rzedowski et al. (2004); las ilustraciones correspondientes pueden consultarse en las últimas dos publicaciones, así como en McVaugh y Rzedowski (1965).

Distribución geográfica conocida: Gro., Méx., Mich., Mor., Oax., Pue. 
34. B. grandifolia (Schltdl.) Engl. (Elaphrium occidentale Rose). Componente por lo general bien definido del complejo de $B$. simaruba, distribuido desde el noroeste de México hasta la costa de Guerrero y también en las cuencas del Santiago y del Balsas. Descripción disponible en Toledo Manzur (1982), en Guízar Nolazco y Sánchez Vélez (1991) y en Johnson (1992); las ilustraciones correspondientes pueden consultarse en las últimas dos publicaciones.

Distribución geográfica conocida: Chih., Col., Dgo., Gro., Jal., Méx., Mich., Mor., Nay., Pue., Sin., Son., Zac.

35. B. graveolens (H.B.K.) Triana \& Planch. (Elaphrium? pubescens Schltdl.). Posiblemente se trata de un elemento que no es nativo de la flora de México, aunque existen colectas procedentes de Veracruz, Oaxaca y Chiapas que aparentemente provienen de plantas silvestres. Tiene amplia reputación de ser medicinal y se le cultiva tanto en huertos familiares, como sobre todo en cercas vivas. Descripción disponible en Rzedowski et al. (2004); la ilustración correspondiente puede consultarse en el mismo trabajo y también en Engler (1931).

Existen registros de poblaciones espontáneas de: Chis., Oax., Ver. y además también de Camp., Hgo., Pue., Tab., pero estos últimos provenientes al parecer sólo de plantas cultivadas.

36. B. heliae Rzed. \& Calderón. Endemismo estrecho de la cuenca del río Tehuantepec. Descripción e ilustración disponibles en Rzedowski y Calderón de Rzedowski (2002).

Distribución geográfica conocida: Oax.

37. B. heteresthes Bullock. Especie distribuida desde el sur de Jalisco hasta la Depresión Central de Chiapas, pero al parecer en forma disyunta, pues no se ha colectado en amplios sectores del este de Guerrero y del oeste de Oaxaca. Descripción disponible en Bullock (1937) y en Toledo Manzur (1982); una ilustración puede consultarse en Bullock (1939).

Distribución geográfica conocida: Chis., Col., Gro., Jal., Méx., Mich., Oax.

38. B. hindsiana Benth. (B. rhoifolia (Benth.) I. M. Johnst., Elaphrium goldmanii Rose, E. macdougalii (Rose) Rose). Planta mayormente arbustiva, común en matorrales xerófilos de la península de Baja California y también registrada de algunas localidades costeras de Sonora. Sin embargo, es necesario aclarar sus relaciones con B. epinnata. Descripción disponible en Wiggins (1964) y en Johnson (1992); las ilustraciones correspondientes pueden consultarse en este último trabajo y en Roberts (1989).

Distribución geográfica conocida: B.C., B.C.S., Son. 
39. B. hintonii Bullock. Elemento más bien poco frecuente y común, distribuido desde el este de Michoacán hasta el este de Oaxaca. Descripción disponible en Bullock (1937), en Toledo Manzur (1982) y en Rzedowski et al. (2004); en la primera y tercera publicaciones pueden consultarse también las ilustraciones correspondientes.

Distribución geográfica conocida: Gro., Méx., Mich., Oax.

40. B. infernidialis Guevara \& Rzed. Endemismo del sector occidental de la Depresión del Balsas. Descripción disponible en Guevara y Rzedowski (1980) y en Toledo Manzur (1982); el primer trabajo mencionado viene acompañado de una ilustración.

Distribución geográfica conocida: Gro., Jal., Mich.

41. B. instabilis McVaugh \& Rzed. Especie de afinidad costera, distribuida de Jalisco a Oaxaca. Descripción disponible en McVaugh y Rzedowski (1965), en Toledo Manzur (1982) y en Pennington y Sarukhán (1998); las ilustraciones correspondientes pueden consultarse en la primera y tercera publicaciones.

Distribución geográfica conocida: Col., Gro., Jal., Mich., Oax.

42. B. isthmica Rzed. \& Calderón. Endemismo estrecho y planta más bien poco frecuente del Istmo de Tehuantepec en el sector cercano al litoral pacífico. Descripción e ilustración disponibles en Rzedowski y Calderón de Rzedowski (2002).

Distribución geográfica conocida: Oax.

43. B. kerberi Engl. (Bursera tecomaca (DC.) Standl. sensu Bullock, no Amyris? tecomaca DC.). Especie frecuente y común en el occidente de México, así como en la porción occidental de la cuenca del Balsas y de la Sierra Madre del Sur. Descripción disponible en Bullock (1939, bajo el nombre de B. tecomaca) y en Toledo Manzur (1982); la primera publicación incluye también una ilustración detallada correspondiente, mientras que la de una hoja puede consultarse en McVaugh y Rzedowski (1965)..

Distribución geográfica conocida: Col., Gro., Jal., Méx., Mich., Nay., Zac.

44. B. krusei Rzed. Elemento hasta hace poco conocido solamente de pocas localidades de la región costera de Guerrero, ahora también se registra de lugares no muy alejados del litoral en el Istmo de Tehuantepec. Descripción e ilustración disponibles en Rzedowski (1973).

Distribución geográfica conocida: Gro., Oax. 
45. B. lancifolia (Schltd.) Engl. (B. trijuga Ramírez). Especie de distribución disyunta. Bastante común en la porción oriental de la cuenca del Balsas y en algunas porciones costeras de Guerrero. También abundante en la cuenca del río Tehuantepec y además presente en el sector nororiental de Querétaro. Descripción disponible en Ramírez (1896, bajo el nombre de B. trijuga), en Toledo Manzur (1982), en Guízar Nolazco y Sánchez Vélez (1991), así como en Rzedowski y Guevara-Féfer (1992); la primera y la tercera publicaciones incluyen también las ilustraciones correspondientes.

Distribución geográfica conocida: Gro., Mor., Oax., Pue., Qro.

46. B. laurihuertae Rzed. \& Calderón. Especie endémica de la cuenca del río Tehuantepec y de la región costera adyacente. Descripción e ilustración disponibles en Rzedowski y Calderón de Rzedowski (2000b).

Distribución geográfica conocida: Oax.

47. B. laxiflora S. Wats. (B. concinna Sandw.). Especie endémica del noroeste de México. Descripción disponible en Wiggins (1965) y en Johnson (1992); en el último trabajo también se ofrece una ilustración.

Distribución geográfica conocida: B.C.S., Chih., Sin., Son.

48. B. linanoe (La Llave) Rzed., Calderón \& Medina. (B. aloexylon Schiede ex Schltdl.) Engl., B. delpechiana Poisson ex Engl., B. longipedunculata (Rose) Standl.). Especie propia del sector oriental de la depresión del Balsas y también de las cuencas del Papaloapan y del río Tehuantepec, donde prospera en altitudes inferiores a $1400 \mathrm{~m}$. Es el linaloe, cuyo aceite esencial se ha exportado intensamente para la industria de los perfumes y que se utiliza aún en la artesanía. Durante muchos años este taxon se ha conocido con los nombres de $B$. aloexylon y de $B$. delpechiana. Descripción disponible en Toledo Manzur (1982), en Guízar Nolazco y Sánchez Vélez (1991) y en Rzedowski et al. (2004). En los dos últimos trabajos se incluye también la ilustración correspondiente.

Distribución geográfica conocida: Gro., Mor., Oax., Pue.

49. B. longicuspis Lundell. Especie descrita de Chiapas, perteneciente al complejo de $B$. simaruba. Plantas de aspecto similar se han colectado también a lo largo del litoral del Pacífico, de Jalisco a Oaxaca, pero es necesario realizar un detallado estudio de todas las poblaciones con el objeto de determinar si efectivamente pertenecen a esta especie. Descripción disponible en Lundell (1961).

Distribución geográfica conocida: Chis. 
50. B. longipes (Rose) Standl. Componente común y bien definido del complejo de $B$. simaruba, restringido en su distribución al sector oriental de la cuenca del Balsas. Descripción disponible en Toledo Manzur (1982) y en Guízar Nolazco y Sánchez Vélez (1991). El último trabajo incluye la ilustración correspondiente.

Distribución geográfica conocida: Gro., Mor., Oax., Pue.

51. B. macvaughiana Cuevas \& Rzed. Endemismo estrecho del sur de Jalisco y de áreas vecinas de Colima. Descripción e ilustración disponibles en Cuevas y Rzedowski (1999).

Distribución geográfica conocida: Col., Jal.

52. B. martae Jiménez Ramírez \& Cruz-Durán. Endemismo estrecho de la parte central de Guerrero, en la región correspondiente a la Sierra Madre del Sur. Descripción e ilustración disponibles en Jiménez Ramírez y Cruz-Durán (2001).

Distribución geográfica conocida: Gro.

53. B. medranoana Rzed. \& Ortiz. Especie de origen híbrido, sólo conocida de la Barranca de Tolantongo, en la parte central de Hidalgo. Descripción e ilustración disponibles en Rzedowski y Ortiz (1988).

Distribución geográfica conocida: Hgo.

54. B. microphylla A. Gray. Elemento restringido en su distribución al noroeste de México (así como a algunas áreas adyacentes de los Estados Unidos). Elemento frecuente de matorrales xerófilos, con frecuencia desarrollándose a manera de arbusto. Descripción disponible en Wiggins (1965) y en Johnson (1992). Las ilustraciones correspondientes pueden consultarse en el último trabajo y en el de Roberts (1989).

Distribución geográfica conocida: B.C., B.C.S., Son.

55. B. mirandae C. A. Toledo. Especie poco frecuente, originalmente sólo conocida de la parte central de Guerrero, también se ha registrado de Puebla y de Oaxaca, en este último estado tanto en la cuenca del Papaloapan, como en la del río Tehuantepec. Descripciones e ilustraciones disponibles en Toledo Manzur (1982, 1984) y en Rzedowski et al. (2004).

Distribución geográfica conocida: Gro., Oax., Pue.

56. B. morelensis Ramírez. Especie de distribución disyunta. Común en el sector oriental de la Depresión del Balsas, apareciendo también en la cuenca del Papaloapan y en la del río Tehuantepec. Además presente en los cañones de los afluentes del Pánuco. Descripción e ilustración disponibles en Ramírez (1896), en 
Toledo Manzur (1982), en Guízar Nolazco y Sánchez Vélez (1991), en Rzedowski y Guevara-Féfer (1992) y en Rzedowski et al. (2004). En la primera, tercera y quinta publicaciones se ofrecen asimismo las ilustraciones correspondientes.

Distribución geográfica conocida: Gto., Gro., Hgo., Mor., Oax., Pue., Qro., S.L.P.

57. B. multifolia (Rose) Engl. Planta sólo conocida de la colección original realizada en el extremo occidental de Zacatecas. También se ha citado de Michoacán, pero las colectas correspondientes pertenecen al parecer a una especie diferente que no se ha dado a conocer todavía. Descripción escueta disponible en Rose (1906, 1911).

Distribución geográfica conocida: Zac.

58. B. multijuga Engl. (B. pringlei S. Wats., B. rubra (Rose) Riley). Especie frecuente y a menudo común en el occidente de México. Descripción disponible en Engler (1883); para la ilustración puede consultarse el trabajo de McVaugh y Rzedowski (1965).

Distribución geográfica conocida: Col., Dgo., Jal., Mich., Nay., Sin., Zac.

59. B. occulta $\mathrm{McV}$. \& Rzed. Endemismo estrecho del extremo occidental de la Sierra Madre del Sur. Descripción e ilustración disponibles en McVaugh y Rzedowski (1965).

Distribución geográfica conocida: Mich.

60. B. palaciosii Rzed. \& Calderón. Endemismo estrecho de la región costera del sur de Jalisco. Descripción e ilustración disponibles en Rzedowski y Calderón de Rzedowski (2000a).

Distribución geográfica conocida: Jal.

61. B. palmeri S. Wats. (Elaphrium queretarense Rose). Especie frecuente del centro-occidente de México, donde habita en altitudes superiores a $1400 \mathrm{~m}$. Algunas de sus poblaciones no se distinguen claramente de las de B. excelsa. Descripción disponible en Rzedowski y Guevara-Féfer (1992).

Distribución geográfica conocida: Ags., Dgo., Gto., Jal., Mich., Qro., Zac.

62. B. paradoxa Guevara \& Rzed. Endemismo estrecho de la región limítrofe entre Guerrero y Michoacán, donde habita en altitudes inferiores a $550 \mathrm{~m}$. Árbol notable por sus foliolos linear-filiformes. Descripción disponible en Guevara-Féfer y Rzedowski (1980), y en Toledo Manzur (1982); en el primer trabajo puede consultarse la ilustración correspondiente.

Distribución geográfica conocida: Gro., Mich. 
63. B. penicillata (DC.) Engl. (B. inopinata Bullock). Elemento frecuente en el occidente de México, extendiéndose a lo largo de la Sierra Madre Occidental hasta Sonora y Chihuahua. Planta cercanamente emparentada con B. graveolens. Descripción disponible en Bullock (1937, bajo el nombre de B. inopinata) y en Rzedowski y Guevara Féfer (1992). Para una ilustración puede consultarse el trabajo de McVaugh y Rzedowski (1965).

Distribución geográfica conocida: Ags., Chih., Col., Dgo., Gto., Jal., Mich., Nay., Sin., Son., Zac.

64. B. pontiveteris Rzed., Calderón \& Medina. Endemismo de la cuenca superior del Papaloapan, donde no es planta frecuente. Descripción e ilustración disponibles en Rzedowski et al. (2004).

Distribución geográfica conocida: Oax., Pue.

65. B. ribana Rzed. \& Calderón. Especie de distribución restringida a la franja costera de Jalisco y de Michoacán, probablemente también presente en Colima. Descripción e ilustración disponibles en Rzedowski y Calderón de Rzedowski (2000a).

Distribución geográfica conocida: Jal., Mich.

66. B. rzedowskii C. A. Toledo. Endemismo estrecho del norte de Guerrero. Descripción e ilustración disponibles en Toledo Manzur (1984).

Distribución geográfica conocida: Gro.

67. B. sarcopoda P. G. Wilson. Especie esencialmente distribuida a lo largo de la Sierra Madre del Sur, desde el sur de Jalisco hasta Oaxaca. En función de las características de sus granos de polen fue transferida a Commiphora, pero estudios más recientes, basados en la secuencia de ADN, indican que debe permanecer como componente de Bursera. Descripción disponible en Wilson (1958) y en Toledo Manzur (1982).

Distribución geográfica conocida: Col., Gro., Jal., Mich., Oax.

68. B. sarukhanii Guevara \& Rzed. Endemismo de la región limítrofe entre Guerrero y Michoacán. Descripción disponible en Guevara-Féfer y Rzedowski (1980), así como en Toledo Manzur (1982). En la primera publicación también puede consultarse la ilustración correspondiente.

Distribución geográfica conocida: Gro., Mich.

69. B. schlechtendalii Engl. (B. jonesii Rose). Especie esencialmente arbustiva, de amplia distribución; común y frecuente en varios sectores de Oaxaca, 
en otros estados más bien de presencia esporádica. Descripción disponible en Toledo Manzur (1982), en Guízar Nolazco y Sánchez Vélez (1991), en Rzedowski y Guevara-Féfer (1992) y en Rzedowski et al. (2004); en las últimas tres publicaciones puede consultarse también la ilustración correspondiente. Además de los estados en seguida mencionados, es factible también su presencia en Ags., Gto., Méx., Mich., Nay. y N.L.

Distribución geográfica conocida: Camp., Chis., Coah., Col., Gro., Hgo., Jal., Mor., Oax., Pue., Qro., S.L.P., Tamps., Yuc., Zac.

70. B. simaruba (L.) Sarg. (B. gummifera L.). Especie de amplia distribución en ambas vertientes de México, a menudo componente de bosques tropicales subcaducifolios y perennifolios en altitudes inferiores a $1200 \mathrm{~m}$. Es la especie más ampliamente distribuida del género, pues se conoce desde el norte de México y el sureste de Estados Unidos, a través de las Antillas y de Centroamérica, hasta el norte de Brasil. No está clara aún su circunscripción y no se entienden bien sus variantes morfológicas. Es el elemento central de un complejo de taxa, varios de los cuales están también pendientes de comprenderse y definirse mejor. Descripción disponible en Toledo Manzur (1982), Rzedowski y Guevara-Féfer (1992), Rzedowski y Calderón de Rzedowski (1996b) y en Rzedowski et al. (2004); en la última publicación puede consultarse el dibujo correspondiente.

Distribución geográfica conocida: Camp., Chis., Col., Dgo., Gto., Gro., Hgo., Jal., Mich., Nay., Oax., Pue., Qro., Q.R., S.L.P., Sin., Son., Tab., Tamps., Ver., Yuc.

71. B. staphyleoides $\mathrm{McV}$. \& Rzed. Endemismo estrecho del sector occidental de la Depresión del Balsas. Componente del complejo de B. fagaroides, cercanamente emparentado con $B$. palaciosii. Descripción e ilustración disponibles en McVaugh y Rzedowski (1965).

Distribución geográfica conocida: Mich.

72. B. stenophylla Sprague \& Riley. Especie emparentada con B. bipinnata y considerada por algunos autores como sinónimo de esta última. Elemento endémico del sector norte de la Sierra Madre Occidental. Descripción disponible en Riley (1923) y en Johnson (1992); en la segunda publicación puede consultarse la ilustración correspondiente.

Distribución geográfica conocida: Chih., Dgo., Sin., Son.

73. B. submoniliformis Engl. Planta común en el sector central y oriental de la cuenca del Balsas y también en la del Papaloapan. Descripción disponible en Toledo Manzur (1982), en Guízar Nolazco y Sánchez Vélez (1991) y en Rzedowski et al. (2004); los dos últimos trabajos incluyen la ilustración correspondiente. 
Distribución geográfica conocida: Gro., Mich., Mor., Oax., Pue. Probablemente también existe en Méx.

74. B. subtrifoliata (Rose) Standl. Planta del grupo de B. fagaroides y quizás sólo una variante de esta última especie. Únicamente conocida del extremo septentrional de Jalisco. Descripción escueta disponible en Rose (1906, 1911); una ilustración puede consultarse en McVaugh y Rzedowski (1965).

Distribución geográfica conocida: Jal.

75. B. suntui C. A. Toledo. Especie endémica del sector oriental de la Depresión del Balsas. Descripción e ilustración disponibles en Toledo Manzur (1984). Distribución geográfica conocida: Gro.

76. B. tecomaca (DC.) Standl. Estrecho endemismo del sector central de la Sierra Madre del Sur en Guerrero. En vista de diferencias en la morfología de sus granos de polen, esta especie fue transferida por Rzedowski y Palacios (1984) al género Commiphora. Sin embargo, recientes estudios basados en el análisis de secuencias de ADN (Becerra y Venable, 1999; Becerra, 2003) indican que su ubicación correcta está en Bursera. Descripción disponible en Toledo Manzur (1982); la ilustración correspondiente puede consultarse en McVaugh y Rzedowski (1965).

Distribución geográfica conocida: Gro.

77. B. trifoliolata Bullock. Especie endémica del sector occidental de la Depresión del Balsas. Componente bien diferenciado del complejo de B. fagaroides. Descripción disponible en Bullock (1936) y en Toledo Manzur (1982).

Distribución geográfica conocida: Gro., Méx., Mich.

78. B. trimera Bullock. Endemismo del sector occidental de la Depresión del Balsas. Descripción disponible en Bullock (1936) y en Toledo Manzur (1982); la ilustración correspondiente puede consultarse en Bullock (1939).

Distribución geográfica conocida: Gro., Jal., Méx., Mich.

79. B. vazquezyanesii Rzed. \& Calderón. Endemismo estrecho de la región cercana al Cabo Corrientes en la costa de Jalisco. Descripción e ilustración disponibles en Rzedowski y Calderón de Rzedowski (2000a).

Distribución geográfica conocida: Jal.

80. B. vejarvazquezii Miranda. Especie endémica del sector oriental de la Depresión del Balsas. Descripción disponible en Miranda (1942), en Toledo Manzur 
(1982) y en Guízar Nolazco y Sánchez Vélez (1991); en la primera y en la última publicación puede consultarse la ilustración correspondiente.

Distribución geográfica conocida: Gro., Mor., Oax., Pue.

81. B. velutina Bullock. Especie de distribución restringida al sector occidental de la cuenca del Balsas, cercanamente emparentada con $B$. copallifera. Descripción disponible en Bullock (1936) y en Toledo Manzur (1982).

Distribución geográfica conocida: Gro., Méx., Mich.

82. B. xochipalensis Rzed. Especie endémica del sector central y oriental de la Depresión del Balsas. Descripción disponible en Rzedowski (1973) y en Toledo Manzur (1982); en la primera publicación puede consultarse la ilustración correspondiente.

Distribución geográfica conocida: Gro., Oax., Pue.

\section{DIVERSIDAD REGIONAL}

De los datos relativos a la distribución geográfica, incluidos en el inciso anterior, se pueden construir el Cuadro 1 y la Figura 1, en los que se define la cantidad y se ofrece una relación de las especies de Bursera, cuya presencia se conoce con cierto margen de seguridad para cada uno de los estados de la República.

Esta información confirma los siguientes hechos importantes ya conocidos previamente:

a) la existencia de Bursera en todos los estados de la República, con excepción de Tlaxcala;

b) la concentración de la diversidad de este conjunto de plantas en las áreas de altitud media y baja de la vertiente pacífica de México y en particular en la cuenca del río Balsas.

Se pone también claramente de manifiesto que:

a) el estado de Guerrero es el territorio más rico en términos de la variedad conocida de componentes de Bursera; le siguen en importancia Michoacán, así como Oaxaca, y a mayor distancia Jalisco y Puebla;

b) el alto número de especies registradas de Oaxaca y Puebla es el resultado de exploraciones y estudios realizados en tiempos relativamente recientes.

Es claro que para la mayor parte de los estados los números indicados no son los definitivos pues, además de los taxa todavía no registrados o desconocidos, faltó incluir otros evidentemente presentes, pero de cuya identidad existen dudas. Sin embargo, las futuras adiciones, así como los cambios taxonómicos y nomenclaturales por venir, probablemente ya no modificarán los lineamientos 
esenciales de la distribución de la diversidad de Bursera en México, tal como se esbozan en la Figura 1, salvo el caso de Oaxaca, para cuyo territorio se espera todavía un aumento tan substancial como para acercarse o igualar la diversidad de Guerrero.

Cuadro 1. Las especies de Bursera registradas de los estados de la República Mexicana.

\begin{tabular}{|c|c|c|}
\hline Ags. & 5 & T. acuminata, B. bipinnata, $B$. fagaroides, $B$. palmeri, $B$. penicillata. \\
\hline B.C. & 4 & B. epinnata, B. fagaroides, B. hindsiana, B. microphylla. \\
\hline B.C.S. & 7 & $\begin{array}{l}\text { B. cerasifolia, B. epinnata, B. fagaroides, B. filicifolia, B. hindsiana, } \\
\text { B. laxiflora, B. microphylla. }\end{array}$ \\
\hline Camp. & 2 & B. schlechtendalii, B. simaruba. \\
\hline Chis. & 8 & $\begin{array}{l}\text { B. ariensis, B. bipinnata, B. excelsa, B. graveolens, B. heteresthes, } \\
\text { B. longicuspis, B. schlechtendalii, B. simaruba. }\end{array}$ \\
\hline Chih. & 6 & $\begin{array}{l}\text { B. fagaroides, B. fragilis, B. grandifolia, B. laxiflora, B. penicillata, } \\
\text { B. stenophylla. }\end{array}$ \\
\hline Coah. & 2 & B. fagaroides, B. schlechtendalii. \\
\hline Col. & 20 & $\begin{array}{l}\text { T. acuminata, B. arborea, B. bipinnata, B. citronella, B. copallifera, } \\
\text { B. denticulata, B. discolor, B. epinnata, B. excelsa, B. fagaroides, } \\
\text { B. grandifolia, B. heteresthes, B. instabilis, B. kerberi, } \\
\text { B. macvaughiana, B. multijuga, B. penicillata, B. sarcopoda, } \\
\text { B. schlechtendalii, B. simaruba. }\end{array}$ \\
\hline D.F. & 2 & B. cuneata, B. fagaroides. \\
\hline Dgo. & 12 & $\begin{array}{l}\text { B. arborea, B. attenuata, B. bipinnata, B. excelsa, B. fagaroides, } \\
\text { B. fragilis, B. grandifolia, B. multijuga, B. palmeri, B. penicillata, } \\
\text { B. simaruba, B. stenophylla. }\end{array}$ \\
\hline Gto. & 8 & $\begin{array}{l}\text { B. bipinnata, B. cuneata, B. fagaroides, B. galeottiana, } \\
\text { B. morelensis, B. palmeri, B. penicillata, B. simaruba. }\end{array}$ \\
\hline Gro. & 48 & $\begin{array}{l}\text { T. acuminata, B. aptera, B. arborea, B. ariensis, B. bicolor, } \\
\text { B. bipinnata, B. bolivarii, B. bonetii, B. chemapodicta, B. citronella, } \\
\text { B. copallifera, B. coyucensis, B. crenata, B. cuneata, B. denticulata, } \\
\text { B. discolor, B. excelsa, B. fagaroides, B. fragrantissima, B. galeottiana, } \\
\text { B. glabrifolia, B. grandifolia, B. heteresthes, B. hintonii, } \\
\text { B. infernidialis, B. instabilis, B. kerberi, B. krusei, B. lancifolia, } \\
\text { B. linanoe, B. longipes, B. martae, B. mirandae, B. morelensis, } \\
\text { B. paradoxa, B. rzedowskii, B. sarcopoda, B. sarukhanii, } \\
\text { B. schlechtendalii, B. simaruba, B. submoniliformis, B. suntui, } \\
\text { B. tecomaca, B. trifoliolata, B. trimera, B. vejarvazquezii, B. velutina, } \\
\text { B. xochipalensis. }\end{array}$ \\
\hline
\end{tabular}


Cuadro 1. Continuación

\begin{tabular}{|c|c|c|}
\hline Hgo. & 6 & $\begin{array}{l}\text { B. cuneata, B. fagaroides, B. medranoana, B. morelensis, } \\
\text { B. schlechtendalii, B. simaruba. }\end{array}$ \\
\hline Jal. & 28 & $\begin{array}{l}\text { T. acuminata, B. arborea, B. ariensis, B. bipinnata, B. citronella, } \\
\text { B. confusa, B. copallifera, B. crenata, B. denticulata, B. excelsa, } \\
\text { B. fagaroides, B. grandifolia, B. heteresthes, B. infernidialis, } \\
\text { B. instabilis, B. kerberi, B. macvaughiana, B. multijuga, } \\
\text { B. palaciosii, B. palmeri, B. penicillata, B. ribana, B. sarcopoda, } \\
\text { B. schlechtendalii, B. simaruba, B. subtrifoliata, B. trimera, } \\
\text { B. vazquezyanesii. }\end{array}$ \\
\hline Méx. & 16 & $\begin{array}{l}\text { T. acuminata, B. ariensis, B. bicolor, B. bipinnata, B.copallifera, } \\
\text { B. cuneata, B. discolor, B. fagaroides, B.glabrifolia, B. grandifolia, } \\
\text { B. heteresthes, B. hintonii, B. kerberi, B. trifoliolata, B. trimera, } \\
\text { B. velutina. }\end{array}$ \\
\hline Mich. & 37 & $\begin{array}{l}\text { T. acuminata, B. arborea, B. ariensis, B. bicolor, B. bipinnata, } \\
\text { B. citronella, B. confusa, B. copallifera, B. coyucensis, B. crenata, } \\
\text { B. cuneata, B. denticulata, B. discolor, B. excelsa, B. fagaroides, } \\
\text { B. fragrantissima, B. glabrifolia, B. grandifolia, B. heteresthes, } \\
\text { B. hintonii, B. infernidialis, B. instabilis, B. kerberi, B. multijuga, } \\
\text { B. occulta, B. palmeri, B. paradoxa, B. penicillata, B. ribana, } \\
\text { B. sarcopoda, B. sarukhanii, B. simaruba, B. staphyleoides, } \\
\text { B. submoniliformis, B. trifoliolata, B. trimera, B. velutina. }\end{array}$ \\
\hline Mor. & 17 & $\begin{array}{l}\text { B. aptera, B. ariensis, B. bicolor, B. bipinnata, B. copallifera, } \\
\text { B. cuneata, B. discolor, B. fagaroides, B. glabrifolia, B. grandifolia, } \\
\text { B. lancifolia, B. linanoe, B. longipes, B. morelensis, } \\
\text { B. schlechtendalii, B. submoniliformis, B. vejarvazquezii. }\end{array}$ \\
\hline Nay. & 13 & $\begin{array}{l}\text { T. acuminata, B. arborea, B. ariensis, B. attenuata, B. bipinnata, } \\
\text { B. copallifera, B. excelsa, B. fagaroides, B. grandifolia, B. kerberi, } \\
\text { B. multijuga, B. penicillata, B. simaruba. }\end{array}$ \\
\hline N.L. & 1 & B. fagaroides. \\
\hline Oax. & 37 & $\begin{array}{l}\text { B. altijuga, B. aptera, B. arida, B. ariensis, B. aspleniifolia, } \\
\text { B. bicolor, B. biflora, B. bipinnata, B. bolivarii, B. cinerea, } \\
\text { B. copallifera, B. discolor, B. esparzae, B. excelsa, B. fagaroides, } \\
\text { B. galeottiana, B. glabrifolia, B. graveolens, B. heliae, } \\
\text { B. heteresthes, B. hintonii, B. instabilis, B. isthmica, B. krusei, } \\
\text { B. lancifolia, B. laurihuertae, B. linanoe, B. longipes, B. mirandae, } \\
\text { B. morelensis, B. pontiveteris, B. sarcopoda, B. schlechtendalii, } \\
\text { B. simaruba, B. submoniliformis, B. vejarvazquezii, B. xochipalensis. }\end{array}$ \\
\hline
\end{tabular}


Cuadro 1. Continuación

\begin{tabular}{|c|c|c|}
\hline Pue. & 28 & $\begin{array}{l}\text { B. altijuga, B. aptera, B. arida, B. ariensis, B. aspleniifolia, } \\
\text { B. bicolor, B. biflora, B. bipinnata, B. bolivarii, B. cinerea, } \\
\text { B. copallifera, B. cuneata, B. discolor, B. fagaroides, B. galeottiana, } \\
\text { B. glabrifolia, B. grandifolia, B. lancifolia, B. linanoe, B. longipes, } \\
\text { B. mirandae, B. morelensis, B. pontiveteris, B. schlechtendalii, } \\
\text { B. simaruba, B. submoniliformis, B. vejarvazquezii, B. xochipalensis. }\end{array}$ \\
\hline Qro. & 8 & $\begin{array}{l}\text { B. cuneata, B. fagaroides, B. galeottiana, B. lancifolia, } \\
\text { B. morelensis, B. palmeri, B. schlechtendalii, B. simaruba. }\end{array}$ \\
\hline Q.R. & 1 & B. simaruba. \\
\hline S.L.P. & 4 & B. fagaroides, B. morelensis, B. schlechtendalii, B. simaruba. \\
\hline Sin. & 11 & $\begin{array}{l}\text { B. arborea, B. attenuata, B. excelsa, B. fagaroides, B. fragilis, } \\
\text { B. grandifolia, B. laxiflora, B. multijuga, B. penicillata, } \\
\text { B. simaruba, B. stenophylla. }\end{array}$ \\
\hline Son. & 9 & $\begin{array}{l}\text { B. fagaroides, B. fragilis, B. grandifolia, B. hindsiana, B. laxiflora, } \\
\text { B. microphylla, B. penicillata, B. simaruba, B. stenophylla. }\end{array}$ \\
\hline Tab. & 1 & B. simaruba. \\
\hline Tamps. & 3 & B. fagaroides, B. schlechtendalii, B. simaruba. \\
\hline Tlax. & 0 & ----- \\
\hline Ver. & 5 & B. bipinnata, B. cinerea, B. fagaroides, B. graveolens, B. simaruba. \\
\hline Yuc. & 2 & B. schlechtendalii, B. simaruba. \\
\hline Zac. & 11 & $\begin{array}{l}\text { T. acuminata, B. bipinnata, B. copallifera, B. fagaroides, } \\
\text { B. grandifolia, B. kerberi, B. multifolia, B. multijuga, B. palmeri, } \\
\text { B. penicillata, B. schlechtendalii. }\end{array}$ \\
\hline
\end{tabular}

Estos dos estados convergen en concentrar el mayor número de especies estrechamente endémicas de Bursera y dentro de los límites de cada uno de ellos se localizan un par de áreas de concentración de tales taxa de distribución restringida, como puede apreciarse en la Figura 2.

\section{ELEMENTOS ESTRECHAMENTE ENDÉMICOS}

Como lo muestra el Cuadro 2, de las 82 especies mexicanas de Bursera que se enlistan en esta contribución, 10 se registran de la localidad tipo y sus alrededores 


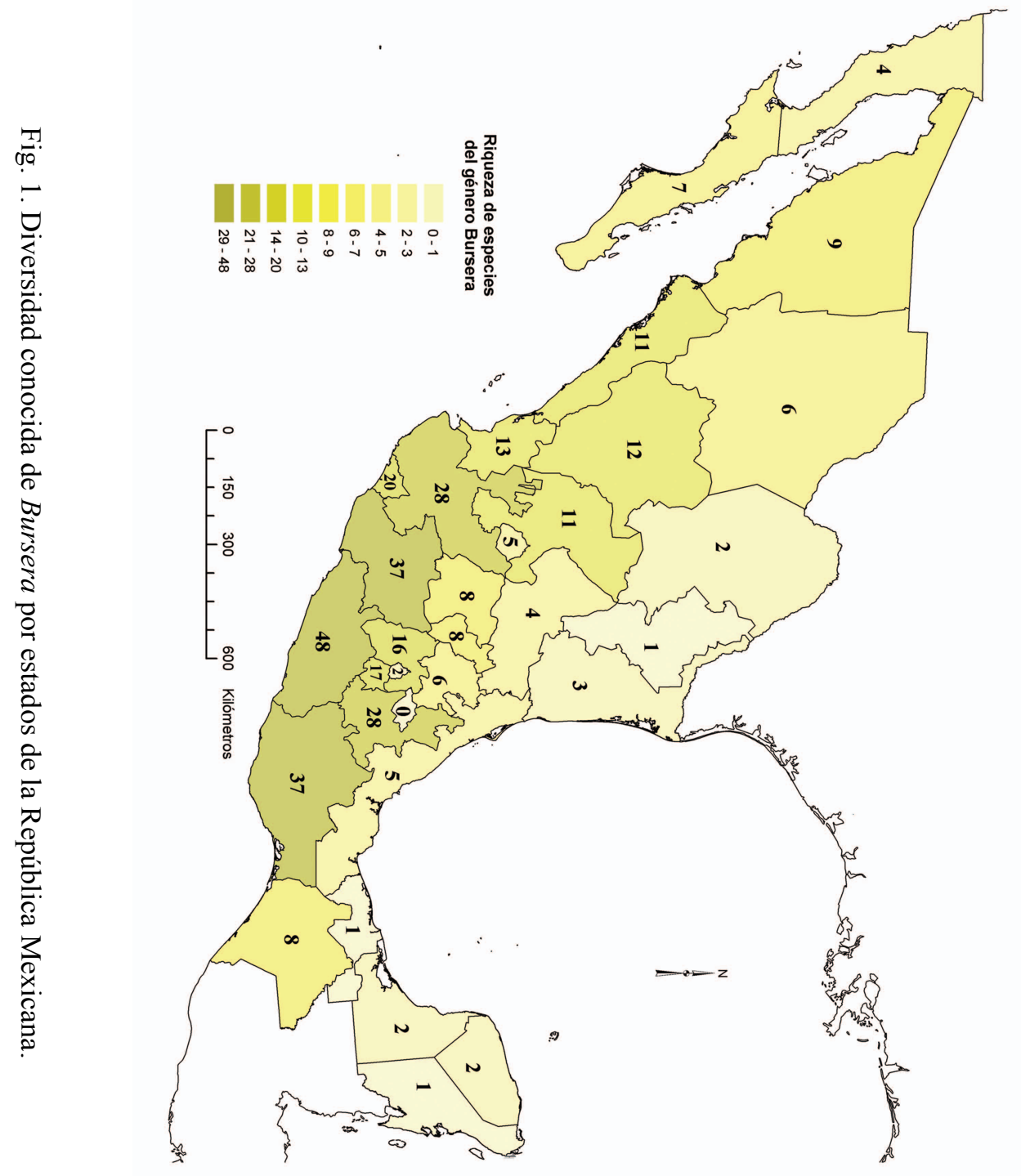


únicamente, 10 adicionales tienen distribución aparentemente restringida a un solo estado, y de acuerdo con el actual conocimiento, el área de otras 13 no se extiende más allá del territorio de dos estados contiguos de la República.

El conjunto de estas 33 especies estrechamente endémicas, representa 40.3\% del total antes señalado para el país, de lo que procede estimar que la proporción real de este grupo probablemente supera $50 \%$, si se toma en cuenta el hecho de que las que están por describirse y por descubrirse deben ser también de distribución limitada, al menos en su gran mayoría.

Esta elevada proporción de endemismos locales apunta claramente hacia el hecho de que el territorio de México ha sido el teatro de una vasta diversificación de Bursera y que en muchos casos las poblaciones actuales de sus especies son de carácter relictual. Los cambios climáticos ocurridos durante el Terciario y el Cuaternario, unidos a la compleja topografía del país, sin duda han propiciado grandes desplazamientos de las comunidades vegetales y con ello estimularon tanto la radiación evolutiva del grupo en cuestión, como también la ulterior reducción de las áreas de distribución y eventual desaparición de muchos de sus representantes. Este aspecto ha sido abordado por Toledo Manzur (1982) quien, entre otras consideraciones, postula la existencia de algunas comarcas que han funcionado como refugios, en los que pudieron sobrevivir especies de Bursera en épocas de condiciones ecológicas desfavorables.

El conocimiento actual revela a su vez la existencia de las siguientes seis áreas de concentración de las especies mexicanas de Bursera de repartición restringida, en las que muy verosímilmente se localizan tales refugios. Su ubicación correspondiente se indica en la Figura 2.

1. Extremo sur de la península de Baja California, de donde se registran $B$. cerasifolia y B. filicifolia.

2. Cinturón costero de Jalisco, Colima y de la porción inmediatamente adyacente de Michoacán, habitat de $B$. palaciosii, B. ribana y B. vazquezyanesii.

3. Sector aledaño al río Balsas, en su recorrido entre Coyuca de Catalán y la presa del Infiernillo, y de ahí a lo largo del valle del río Tepalcatepec, área de crecimiento de B. coyucensis, B. paradoxa, B. sarukhanii y B. staphyleoides.

4. Fracción del centro del estado de Guerrero que incluye la región de Chilpancingo, de Iguala y algunas comarcas adyacentes, es el albergue de $B$. bonetii, B. chemapodicta, B. martae, B. rzedowskii y B. tecomaca.

5. Parte más seca de la cuenca alta del río Papaloapan, con extensión hacia los alrededores de Huajuapan, en el NW de Oaxaca y áreas aledañas de Puebla, donde prosperan $B$. altijuga, $B$. arida, $B$. aspleniifolia y $B$. pontiveteris.

6. Porción media e inferior de la cuenca del río Tehuantepec, así como la región istmeña vecina, en las que se colectaron B. heliae, B. isthmica y B. laurihuertae. 
Acta Botanica Mexicana 70: 85-111 (2005)

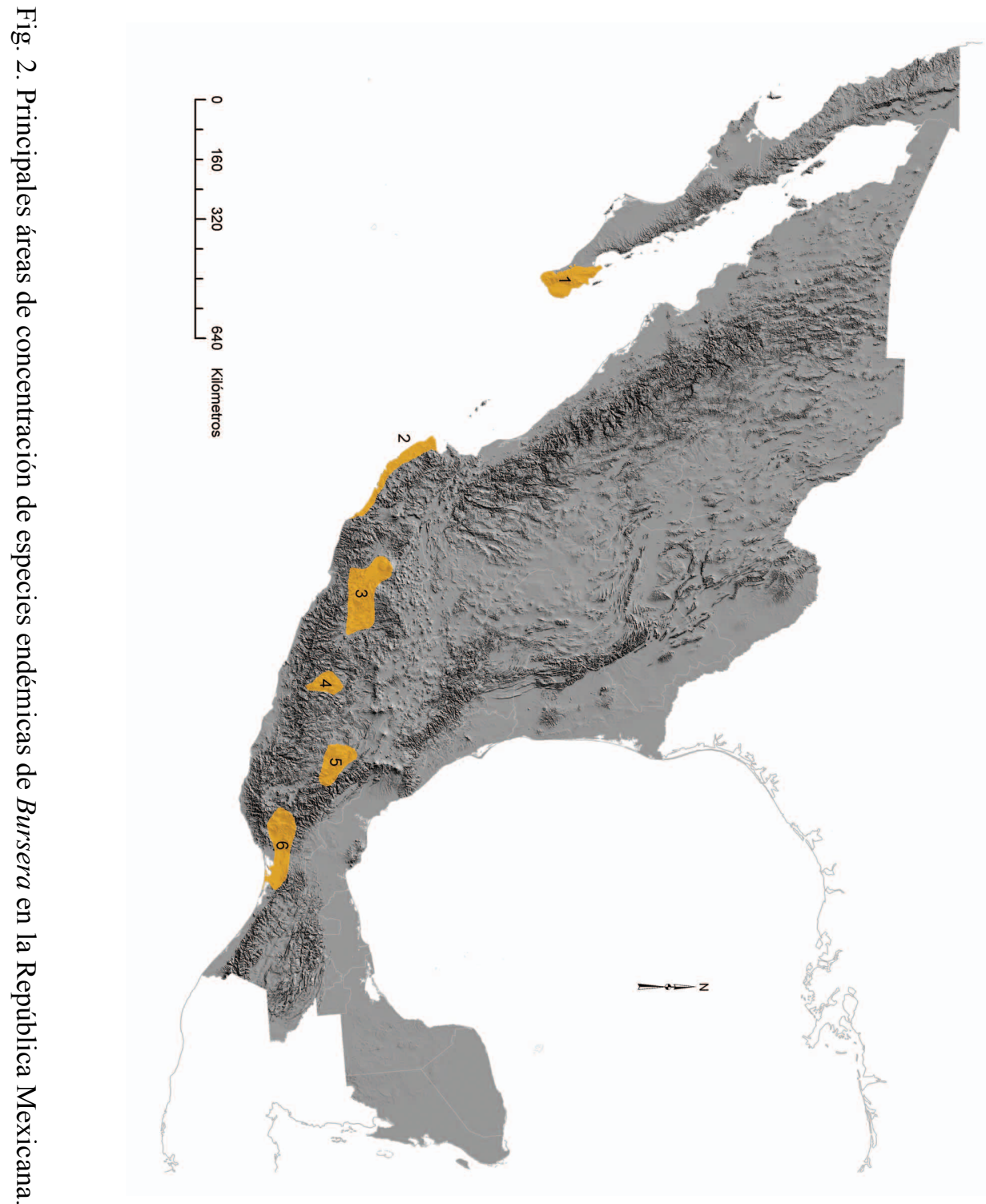


Cuadro 2. Especies mexicanas de Bursera de distribución particularmente restringida.

\begin{tabular}{|l|l|l|}
\hline $\begin{array}{c}\text { Sólo conocidas de la } \\
\text { localidad tipo y de áreas } \\
\text { vecinas }\end{array}$ & \multicolumn{1}{|c|}{$\begin{array}{c}\text { Sólo conocidas de un } \\
\text { estado }\end{array}$} & $\begin{array}{c}\text { Sólo conocidas de dos estados } \\
\text { contiguos }\end{array}$ \\
\hline B. chemapodicta, Gro. & B. bonetii, Gro. & B. altijuga, Oax., Pue. \\
\hline B. longicuspis, Chis. & B. cerasifolia, B.C.S. & B. arida, Oax., Pue. \\
\hline B. martae, Gro. & B. esparzae, Oax. & B. aspleniifolia, Oax., Pue. \\
\hline B. medranoana, Hgo. & B. filicifolia, B.C.S. & B. biflora, Oax., Pue. \\
\hline B. multifolia, Zac. & B. heliae, Oax. & B. confusa, Jal., Mich. \\
\hline B. palaciosii, Jal. & B. isthmica, Oax. & B. coyucensis, Gro., Mich. \\
\hline B. rzedowskii, Gro. & B. laurihuertae, Oax. & B. fragrantissima, Gro., Mich. \\
\hline B. subtrifoliata, Jal. & B. occulta, Mich. & B. krusei, Gro., Oax. \\
\hline B. tecomaca, Gro. & B. staphyleoides, Mich. & B. macvaughiana, Col., Jal. \\
\hline B. vazquezyanesii, Jal. & B. suntui, Gro. & B. paradoxa, Gro., Mich. \\
\hline & & B. pontiveteris, Oax., Pue. \\
\hline & & B. ribana, Jal., Mich. \\
\hline & & B. sarukhanii, Gro., Mich. \\
\hline
\end{tabular}

Cabe agregar que para las áreas 1, 2, 3, 4 y 6 se tiene apuntada la presencia de especies adicionales, aún no dadas formalmente a conocer.

\section{LITERATURA CITADA}

Becerra, J. X. 2003. Evolution of Mexican Bursera (Burseraceae) inferred from ITS, ETS, and 5S nuclear ribosomal DNA sequences. Molec. Phylogen. Evol. 26: 300-309.

Becerra, J. X. \& D. L. Venable. 1999. Nuclear ribosomal DNA phylogeny and its implications for evolutionary trends in Mexican Bursera (Burseraceae). Amer. Journ. Bot. 86: 1047-1057.

Bullock, A. A. 1936. Notes on the Mexican species of Bursera. Kew Bull. 1936: 346-387.

Bullock, A. A. 1937-1938. Further notes on the genus Bursera. Kew Bull. 1937: 447-458; 1938 : 163-168.

Bullock, A. A. 1939. Bursera hintoni Bullock, B. trimera Bullock, B. heteresthes Bullock \& B. tecomaca (DC.) Standl. Hooker's Ic. P1. 34, tt. 3392-3395.

Cuevas G., R. y J. Rzedowski. 1999. Una especie nueva de Bursera (Burseraceae) del occidente de México. Acta Bot. Mex. 46: 77-81.

Engler, A. 1883. Burseraceae. DC. Monogr. Phaner. 4: 1-169.

Engler, A. 1931. Burseraceae. In: Engler, A. \& K. Prantl. Die natürlichen Pflanzenfamilien. ed. 2. 19a: 405-456. 
Guevara-Féfer, F. y J. Rzedowski. 1980. Notas sobre el género Bursera (Burseraceae) en Michoacán (México). I. Tres especies nuevas de los alrededores de la presa del Infiernillo, con algunos datos relativos a la región. Bol. Soc. Bot. Méx. 39: 63-81.

Guízar Nolazco, E. y A. Sánchez Vélez. 1991. Guía para el reconocimiento de los principales árboles del alto Balsas. Universidad Autónoma Chapingo. Chapingo, Méx. 207 pp.

Humboldt, A., A. Bonpland \& K. S. Kunth. 1825. Burseraceae, In: Nova genera et species plantarum 7: 23-35, tt. 611, 612.

Jiménez Ramírez, J. y R. Cruz Durán. 2001. Una especie nueva de Bursera (Burseraceae) del estado de Guerrero, México. Acta Bot. Mex. 55: 7-12.

Johnson, M. B. 1992. The genus Bursera in Sonora, Mexico and Arizona, U.S.A. Desert Plants 10: 126-143.

Kohlmann, B. y S. Sánchez. 1984. Estudio areográfico del género Bursera Jacq. ex L. (Burseraceae) en México: una síntesis de métodos. In: Ezcurra, E. et al. Métodos cuantitativos en la biogeografía. Instituto de Ecología, A.C. Publicación No. 12. México, D.F. pp. 45-125.

Lundell, C. L. 1961. Plantae mayanae. IV. Wrightia 3: 1-20.

McVaugh, R. \& J. Rzedowski. 1965. Synopsis of the genus Bursera L. in western Mexico, with notes on the material of Bursera collected by Sessé \& Mociño. Kew Bull. 18: 317-382.

Miranda, F. 1942. Nuevas fanerógamas del S.O. del estado de Puebla. An. Inst. Biol. Méx. 13: 451-462.

Pennington, T. D. y J. Sarukhán. 1998. Árboles tropicales de México. 2a. ed. Universidad Nacional Autónoma de México y Fondo de Cultura Económica. México, D.F. 521 pp.

Ramírez, J. 1896. Datos para el estudio de los cuajiotes. An. Inst. Med. Nac. 2: 14-18.

Riley, L. A. M. 1923. Contributions to the flora of Sinaloa. Kew Bull. Misc. Inf. 4: 163-175.

Roberts, N. C. 1989. Baja California plant field guide. Natural History Publishing Company. La Jolla, Calif. 309 pp.

Rose, J. N. 1906. Studies of Mexican and Central American plants. 5. Contr. U.S. Nat. Herb. 10: 79-132.

Rose, J. N. 1909. Studies of Mexican and Central American plants. 6. Contr. U. S. Nat. Herb. 12: 259-302.

Rose, J. N. 1911. Burseraceae. North Amer. Fl. 25: 241-261.

Rzedowski, J. 1968. Notas sobre el género Bursera (Burseraceae) en el estado de Guerrero. An. Esc. Nac. Cienc. Biol. Méx. 17: 17-36.

Rzedowski, J. 1978. Plantae Guerrerenses Kruseanae. Ciencia, Méx. 28: 49-56.

Rzedowski, J. y G. Calderón de Rzedowski. 1996a. Nota sobre Bursera cinerea Engl. (Burseraceae) en el estado de Veracruz. Acta Bot. Mex. 37: 33-38.

Rzedowski, J. y G. Calderón de Rzedowski. 1996b. Burseraceae. Flora de Veracruz 94: 1-37.

Rzedowski, J. y G. Calderón de Rzedowski. 2000a. Tres especies nuevas de Bursera (Burseraceae) de la región costera del occidente de México. Acta Bot. Mex. 50: 4759.

Rzedowski, J. y G. Calderón de Rzedowski. 2000b. Una especie nueva de Bursera (Burseraceae) del estado de Oaxaca (México). Acta Bot. Mex. 52: 75-81. 
Rzedowski, J. y G. Calderón de Rzedowski. 2002. Dos especies nuevas de Bursera (Burseraceae) del estado de Oaxaca (México). Acta Bot. Mex. 59: 81-90.

Rzedowski, J. y F. Guevara-Féfer. 1992. Burseraceae. Flora del Bajío y de Regiones Adyacentes 3: 1-46.

Rzedowski, J., R. Medina Lemos y G. Calderón de Rzedowski. 2004. Las especies de Bursera (Burseraceae) en la cuenca superior del río Papaloapan (México). Acta Bot. Mex. 66: 23-151.

Rzedowski, J. y E. Ortiz. 1982. Estudios quimiotaxonómicos de Bursera (Burseraceae). I. Bursera chemapodicta sp. n. Bol. Soc. Bot. Méx. 43: 73-80.

Rzedowski, J. y E. Ortiz. 1988. Estudios quimiotaxonómicos de Bursera (Burseraceae). II. Una especie nueva de origen híbrido de la Barranca de Tolantongo, estado de Hidalgo. Acta Bot. Mex. 1: 11-19.

Rzedowski, J. y R. Palacios-Chávez. 1985. La presencia de Commiphora (Burseraceae) en México. Taxon 34: 207-210.

Schlechtendal, D. F. L. 1843. De plantis Mexicanis a G. Schiede, M.Dr., Car. Ehrenbergio alliisque, collectis. Linnaea 17: 625-631.

Toledo Manzur, C. A. 1982. El género Bursera (Burseraceae) en el estado de Guerrero. Tesis. Facultad de Ciencias. Universidad Nacional Autónoma de México. México, D.F. 182 pp.

Toledo Manzur, C. A. 1984. Contribuciones a la flora de Guerrero: Tres especies nuevas del género Bursera (Burseraceae). Biotica 9: 441-449.

Wilson, P. G. 1958. Plantae Mexicanae Hintonianae 12. Kew Bull. 1958: 155-170. 